\title{
Secretion of FK506/FK520 and rapamycin by Streptomyces inhibits the growth of competing Saccharomyces cerevisiae and Cryptococcus neoformans
}

\author{
Cristl Arndt, Maria Cristina Cruz, Maria E. Cardenas and Joseph Heitman
}

Author for correspondence: Joseph Heitman. Tel: +1 919684 2824. Fax : + 19196845458. e-mail: heitm001@mc.duke.edu

Departments of Genetics, Pharmacology and Cancer Biology, Microbiology and Medicine, the Howard Hughes Medical Institute, 322 Carl Building, Research Drive, Box 3546 Duke University Medical Center, Durham, NC 27710, USA

\begin{abstract}
FK506 and rapamycin are immunosuppressants that inhibit signalling cascades required for T-cell activation, yet both are natural products of Streptomyces that live in the soil. FK506 and rapamycin also have potent antimicrobial activity against yeast and pathogenic fungi, suggesting a natural role in inhibiting growth of competing micro-organisms. The immunosuppressive and antimicrobial activities of FK506 and rapamycin are mediated by binding to the FKBP12 prolyl isomerase and the resulting FKBP12/FK506 and FKBP12/rapamycin complexes inhibit conserved protein targets, either the phosphatase calcineurin or the TOR (target of rapamycin) kinases, respectively. Streptomyces sp., 'Streptomyces hygroscopicus subsp. ascomyceticus' and Streptomyces hygroscopicus, which produce FK506, FK520 (also known as ascomycin, a C21 ethyl derivative of FK506) and rapamycin, respectively, produced toxins that inhibited the growth of competing cells of the yeast Saccharomyces cerevisiae and the pathogenic fungus Cryptococcus neoformans. Yeast and fungal mutants lacking FKBP12 or expressing dominant drug-resistant calcineurin or TOR mutants were resistant to FK506 and rapamycin, and to the toxins produced by Streptomyces. Streptomyces strains with mutations in the FK506 or rapamycin biosynthetic enzymes were impaired in toxin production. Finally, the toxins secreted by 'S. hygroscopicus subsp. ascomyceticus' and S. hygroscopicus promoted formation of FKBP12/calcineurin and FKBP12/TOR complexes in a two-hybrid assay and mutations that rendered calcineurin or TOR drug-resistant prevented interaction. These observations support the hypothesis that Streptomyces evolved to secrete FK506, FK520 and rapamycin as toxins to inhibit the growth of competing yeast and fungi.
\end{abstract}

Keywords: FK506/FK520, rapamycin, immunosuppression, Streptomyces, growth inhibition

\section{INTRODUCTION}

A variety of micro-organisms produce secondary metabolites or natural products, many of which exhibit toxic activity against different cells and organisms (Chadwick \& Whelan, 1992). These natural products have proved to be a valuable resource to identify drugs with specific physiological effects in animals. For example, the immunosuppressants FK506 (tacrolimus),

Abbreviation: TOR, target of rapamycin.
FK520 (ascomycin, an active FK506 derivative in which the C21 allyl group is replaced by an ethyl group) and rapamycin (sirolimus) were originally identified by screening random soil samples for biological activities and were later found to be products of Streptomyces sp., 'Streptomyces hygroscopicus subsp. ascomyceticus' and Streptomyces hygroscopicus, actinomycetes that live in the soil (Baker et al., 1978; Hatanaka et al., 1988a, b; Kino et al., 1987; Morisaki \& Arai, 1992; Sehgal et al., 1975; Singh et al., 1979; Vezina et al., 1975). FK506 is now in widespread use as an immunosuppressant to prevent and treat graft rejection in solid organ transplant 
recipients and for graft-vs. host disease in bone marrow transplant recipients. Rapamycin is in late phase III clinical trials for similar purposes (Cardenas et al., 1995a).

Considerable progress has been made in delineating the biosynthetic pathways by which Streptomyces elaborates these complex macrolide compounds. A cluster of enzymes has been identified which mediates rapamycin biosynthesis (Schwecke et al., 1995) and mutational approaches have been employed to define the pathways involved in both rapamycin (Khaw et al., 1998; Lomovskaya et al., 1997) and FK506 biosynthesis (Motamedi et al., 1997). Finally, numerous analogues of these compounds have been produced by biotransformation, altered growth medium conditions and semisynthetic organic chemistry (Khaw et al., 1998; Luengo et al., 1995; Nishida et al., 1995), and these analogues might have novel or improved biological activities.

FK506 and rapamycin suppress the immune system by inhibiting signal transduction cascades required for $\mathrm{T}$ lymphocyte activation during tissue rejection. In mammals, the activities of FK506 and rapamycin are relatively specific for lymphocytes, with only limited effects observed in other cells and tissues. This cell type specificity of FK506 and rapamycin raises a paradox: how did soil micro-organisms evolve to produce such $\mathrm{T}$ cell-specific agents when they rarely, if ever, encounter large animals? Moreover, suppressing the immune system is not a particularly effective or rapid means of doing harm to a large animal and many microbial toxins directed against mammals target the nervous system. Hence, immunosuppression by FK506 or rapamycin is not likely to be a normal role in nature. In fact, FK506, cyclosporin $\mathrm{A}$ and rapamycin exhibit antimicrobial activity against a variety of different bacteria, yeasts, parasites and fungi and might therefore function to inhibit the growth of competing micro-organisms (High, 1994; McCabe et al., 1986; Odom et al., 1997a, b; Wong et al., 1998).

Recent studies reveal that the molecular mechanisms of the immunosuppressive and antimicrobial effects of FK506 and rapamycin are remarkably similar and involve inhibition of highly conserved target proteins (Abraham \& Wiederrecht, 1996; Heitman et al., 1992; Schreiber \& Crabtree, 1992). Both drugs are hydrophobic and enter the cell by diffusion. Next they bind to a highly conserved intracellular protein, FKBP12, which is conserved from unicellular eukaryotes such as Saccharomyces cerevisiae to man. FKBP12 is an enzyme that catalyses cis-trans peptidyl prolyl isomerization, a rate-limiting step during protein refolding in vitro. Both FK506 and rapamycin bind to the FKBP12 active site and inhibit activity, but this is not the mechanism by which these agents are toxic. Instead, the protein/drug complexes are the active in vivo agents. For example, yeast mutants lacking FKBP12 are viable and resistant to FK506 and rapamycin (Breuder et al., 1994; Heitman et al., 1991a, b; Hemenway et al., 1995; Parent et al., 1993) and increasing FKBP12 protein levels in mammalian cells by transfection results in increased drug sensitivity (Bram et al., 1993).

The targets of FKBP12/drug complexes are elements of signal transduction pathways conserved from yeast to man. The target of the FKBP12/FK506 complex was first identified as calcineurin by drug affinity chromatography of mammalian cell extracts (Liu et al., 1991). Subsequent studies revealed that calcineurin was the conserved target of FK506 in yeast (Breuder et al., 1994; Foor et al., 1992; Parent et al., 1993). Calcineurin is a calcium/calmodulin activated protein phosphatase that plays a central role in signal transduction from the T-cell receptor to regulate $\mathrm{T}$-cell activation and plays diverse roles in yeast involving gene regulation and cation homeostasis. The target of the FKBP12/rapamycin complex was first identified by genetic and molecular studies in yeast as the TOR (target of rapamycin) kinase homologues (Cafferkey et al., 1993; Heitman et al., 1991a, b; Helliwell et al., 1994; Kunz et al., 1993) and the mammalian target of rapamycin (mTOR, RAFT1, FRAP) shares $\sim 50 \%$ sequence identity with yeast TOR proteins (Brown et al., 1994; Chiu et al., 1994; Sabatini et al., 1994; Sabers et al., 1995). TOR kinase has been functionally conserved from yeast to man and plays a central role in regulating cell cycle progression and translation in lymphocytes in response to growth factors and, in yeast, in response to nutrients (Alarcon et al., 1996; Barbet et al., 1996; Brunn et al., 1997; di Como \& Arndt, 1996).

Taken together, these observations suggest that Streptomyces may have originally evolved the ability to synthesize and secrete FK506 and rapamycin as antimicrobial agents to inhibit the growth of competing micro-organisms in the soil. Such a role would be analogous to that attributed to antibiotics produced by fungi. Because the proteins that FK506 and rapamycin target have been highly conserved during evolution, their original activity against yeast and fungal targets could have been maintained against the mammalian homologues. Here we have tested this hypothesis and present evidence that Streptomyces spp. indeed secrete potent toxins that inhibit the growth of competing cells of the ascomycetous yeast Saccharomyces cerevisiae and also of the very distantly related basidiomycetous pathogenic fungus Cryptococcus neoformans.

\section{METHODS}

Media. Yeast YPD medium was prepared as described by Sherman (1991). Streptomyces sp. and 'S. hygroscopicus subsp. ascomyceticus' were maintained on yeast malt extract agar (Difco medium 0770) and Streptomyces hygroscopicus on sporulation agar $\left(\mathrm{g} \mathrm{l}^{-1}\right.$ : yeast extract, 1 ; beef extract, 1; tryptose, 2; glucose, 10; agar, 15).

Yeast and bacterial strains. S. bygroscopicus subsp. ascomyceticus' (ATCC 14891, hereafter referred to as ' $S$. ascomyceticus') and S. hygroscopicus (ATCC 29253) were obtained from the American Type Culture Collection, Manassas, VA, USA. Both strains exhibited previously de- 
Table 1. Yeast strains

\begin{tabular}{|c|c|c|}
\hline Strain & Genotype & Reference/source \\
\hline \multicolumn{3}{|c|}{ Saccharomyces cerevisiae } \\
\hline $\mathrm{JK} 9-3 \mathrm{~d} \alpha$ & $\begin{array}{l}\text { MAT } \alpha \text { trp1 his4 leu2-3,112 ura3-52 rme1 } \mathrm{GAL}^{+} \\
\mathrm{HMLa}\end{array}$ & Heitman et al. (1991b) \\
\hline JHY3-3B & $\mathrm{JK} 9-3 \mathrm{~d} \alpha$ fpr $1:: U R A 3-3\left(\mathrm{FK} 506^{\mathrm{R}}\right.$ rapa $\left.^{\mathrm{R}}\right)$ & Heitman et al. (1991b) \\
\hline $\mathrm{R} 1$ & JK9-3d $\alpha$ tor $1-1\left(\mathrm{FK}^{2} 06^{\mathrm{S}} \mathrm{rapa}^{\mathrm{R}}\right)$ & Heitman et al. (1991a) \\
\hline R17 & JK9-3d $\alpha$ tor $2-1$ HIS $^{+}\left(\mathrm{FK} 506^{\mathrm{S}} \mathrm{rapa}^{\mathrm{R}}\right)$ & Heitman et al. (1991a) \\
\hline IL $993 / 5 \mathrm{c}$ & $M A T \alpha \rho^{\circ}$ ilv $5\left(\mathrm{CsA}^{\mathrm{s}} \mathrm{FK} 506^{\mathrm{s}}\right)$ & Tropschug et al. (1989) \\
\hline ТВ23 & IL993/5c ura3 leu2:: hisG (CsAs FK506") & Breuder et al. (1994) \\
\hline $6 \mathrm{~A}$ & IL993/5c ura3 leu2: : hisG CMP2-1 (CsA ${ }^{\mathrm{s}}$ FK506 ${ }^{\mathrm{R}}$ ) & Cardenas et al. (1995b) \\
\hline TB62 & $\begin{array}{l}\text { IL993/5c MATa ura3 ILV } V^{+} \text {fpr1::URA3-3 }\left(\mathrm{CsA}^{\mathrm{s}}\right. \\
\left.\text { FK506 }{ }^{\mathrm{R}}\right)\end{array}$ & Breuder et al. (1994) \\
\hline PJ69-4A & 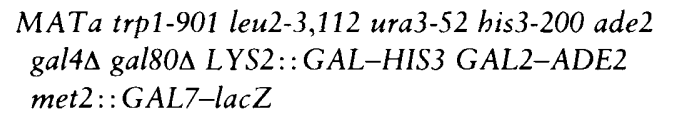 & James et al. (1996) \\
\hline SMY $87-4$ & PJ69-4A fpr1:: hisG & \\
\hline \multicolumn{3}{|c|}{ Cryptococcus neoformans } \\
\hline JEC21 & MAT $\alpha\left(\right.$ FK506 rapa $\left.^{\mathrm{s}}\right)$ & Moore \& Edman (1993) \\
\hline $\mathrm{C} 21 \mathrm{~F} 2$ & $M A T \propto F K R 1-1\left(\mathrm{FK}^{2} 06^{\mathrm{R}} \mathrm{rapa}^{\mathrm{s}}\right)$ & Odom et al. (1997a) \\
\hline $\mathrm{C} 21 \mathrm{~F} 3$ & MAT $\alpha$ frr1-3 (FK506 $\left.{ }^{\mathrm{R}} \mathrm{rapa}^{\mathrm{R}}\right)$ & Odom et al. (1997a) \\
\hline CN111 & MAT $\alpha \operatorname{cyh}^{\mathrm{R}}$ TOR1-1 (FK506 rapa $\left.^{\mathrm{R}}\right)$ & Cruz et al. (1999) \\
\hline
\end{tabular}

scribed morphological characteristics. For example, S. bygroscopicus formed colonies that invaded the agar, were quite hydrophobic and formed colonies of two distinct types, either powdery whitish grey or dark black (Vezina et al., 1975). The isogenic pair of rapamycin-producing (ATCC 29253) and rapamycin-deficient (WHM1651) strains of S. hygroscopicus were kindly provided by Dr C. Hutchinson, University of Wisconsin, Madison, WI, USA (Lomovskaya et al., 1997). The isogenic pair of FK506-producing (MA6548) and FK506reduced production strains of Streptomyces sp. were provided by Dr Haideh Motamedi, Merck, Rahway, NJ, USA (Motamedi et al., 1997).

Saccharomyces cerevisiae and C. neoformans strains are listed in Table 1 and most have been described previously (Breuder et al., 1994; Cardenas et al., 1995b; Heitman et al., 1991a, b; James et al., 1996; Odom et al., 1997a, b). Strain SMY87-4 is an isogenic derivative of the two-hybrid host strain PJ69-4A (James et al., 1996) in which the FKBP12-encoding gene, FPR 1, has been disrupted by fpr1::hisG-URA3-hisG and then selected for $f$ pr $1:$ : his $G$ recombinants by selection for growth on 5-fluoroorotic acid (5-FOA) medium.

Growth inhibition assays. Saccharomyces cerevisiae or $C$. neoformans strains were cultured in YPD liquid medium and $\sim 10^{7}$ cells were resuspended in $3 \mathrm{ml}$ top agar $(0.7 \%$ Bacto agar in water), which was poured onto the surface of YPD medium ( 90 or $140 \mathrm{~mm}$ plates) and allowed to solidify. Discs containing either 'S. ascomyceticus' or S. hygroscopicus were prepared by incubating a lawn of cells on sporulation agar at $24{ }^{\circ} \mathrm{C}$. Plugs of agar with overlaying colonies, mycelium and spores were excised with the larger end of a sterile Pasteur pipette and placed (colony side down) on the surface of medium containing yeast or fungal cells. Co-cultures were incubated for $48 \mathrm{~h}$ at 30 or $37^{\circ} \mathrm{C}$. Growth inhibition was quantified by measuring the distance from the edge of the disc to the zone of growth (the radius of growth inhibition).
Two-hybrid interactions. Plasmids expressing the GAL4(BD)/yeast FKBP12, GAL4(AD)/yeast calcineurin A, GAL4(BD)/C. neoformans TOR1 FKBP12/rapamycin-binding domain and GAL4(AD)/C. neoformans FKBP12 have been described (Cardenas et al., 1994, 1995b; Cruz et al., 1999). A two-hybrid host strain, SMY87-4 (see Table 1), was co-transformed with control plasmids, with plasmids expressing GAL4(BD)/yeast FKBP12 and wild-type and mutant GAL4(AD)/yeast calcineurin fusions, and with plasmids expressing wild-type and mutant GAL4(BD)/TOR1 and GAL4(AD)/FKBP12 fusions. Cells were resuspended in top agar on the surface of synthetic medium lacking tryptophan, leucine and adenine, and were then overlaid with inverted agar discs bearing ' $S$. ascomyceticus' or S. hygroscopicus. Control discs contained FK506 $(1 \mu \mathrm{g})$, rapamycin $(1 \mu \mathrm{g})$ or no drug. Cells were incubated for $3-5 \mathrm{~d}$ at $30^{\circ} \mathrm{C}$. Colony formation is indicative of GAL4-dependent expression of the $G A L-A D E 2$ reporter gene fusion as a consequence of FKBP12/ligand/ calcineurin or FKBP12/ligand/TOR interactions.

\section{RESULTS}

\section{Streptomyces inhibits Saccharomyces cerevisiae growth via FKBP12-dependent inhibition of calcineurin}

To determine if FK506 and FK520-producing Streptomyces spp. secrete toxins that inhibit the growth of competing micro-organisms, we utilized a series of mutants of the yeast Saccharomyces cerevisiae that are sensitive or resistant to FK506 and FK520 by defined genetic mutations. Many laboratory strains of yeast are not sensitive to FK506 or FK520 because the enzyme inhibited by this drug, calcineurin, is not required for vegetative growth. A variety of mutants of Saccharo- 

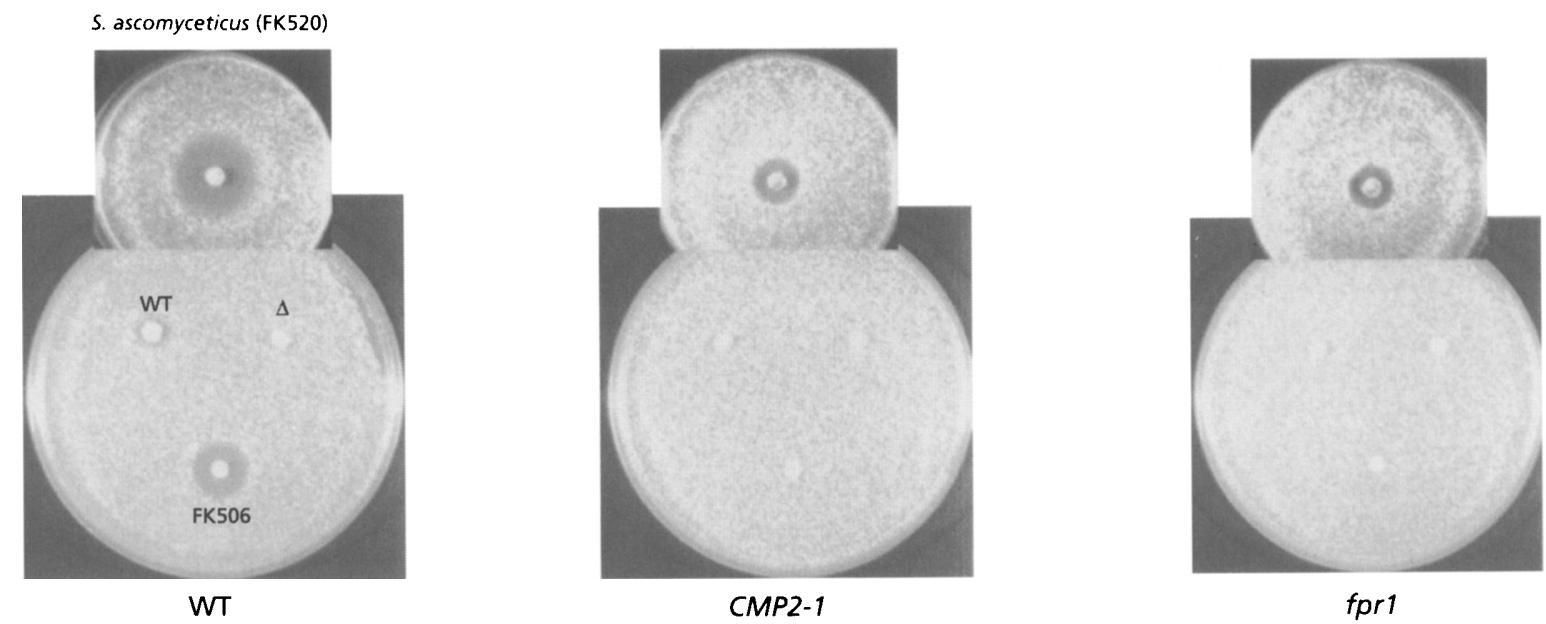

\begin{abstract}
Fig. 1. Streptomyces sp. and 'S. ascomyceticus' inhibit the growth of wild-type Saccharomyces cerevisiae but not of FK506/FK520-resistant mutant strains. Cells of isogenic yeast strains expressing wild-type FKBP12 and calcineurin proteins (WT, TB23), or expressing an FK506/FK520-resistant calcineurin mutant (CMP2-1, 6A), or lacking FKBP12 (fpr1, TB62) were resuspended in top agar on the surface of YPD medium. The medium was then overlaid with inverted agar discs bearing 'S. ascomyceticus' (upper disc), Streptomyces sp. FK506-producing strain MA6548 (WT, left disc), Streptomyces sp. strain with reduced FK506 production ( $\Delta$, right disc) or a control disc with $1 \mu \mathrm{g}$ FK506 (lower disc) and incubated at $30{ }^{\circ} \mathrm{C}$ for $48 \mathrm{~h}$.
\end{abstract}

myces cerevisiae have been identified in which calcineurin is essential for viability and these strains are sensitive to growth inhibition by FK506 and FK520 at a MIC of $\sim 1 \mu \mathrm{g} \mathrm{ml}^{-1}$ (Breuder et al., 1994; Douglas et al., 1994; Hemenway et al., 1995; Parent et al., 1993). One such calcineurin-dependent, FK506/FK520-sensitive yeast strain, TB23, has defects in ion homeostasis that render calcineurin essential (Breuder et al., 1994). Isogenic FK506-resistant mutants derived from strain TB23 lack the FK506-binding protein FKBP12 ( $p p r 1)$ or express dominant FK506-resistant forms of the FKBP12/FK506 target protein calcineurin (CMP2-1) that retain catalytic function but do not bind FKBP12/FK506 (Breuder et al., 1994; Cardenas et al., 1995b).

To establish whether ' $S$. ascomyceticus' secretes the toxin FK520, the FK506- and FK520-sensitive yeast strain TB23 was cultured in the presence of ' $S$. ascomyceticus'. For these experiments, yeast cells were resuspended in molten top agar and overlaid on rich YPD medium. Once the top agar had solidified, an agar disc containing ' $S$. ascomyceticus' was placed on the surface. Growth of the FK520-sensitive yeast strain TB23 was potently inhibited by ' $S$. ascomyceticus', resulting in a zone of inhibition with a radius of $1 \mathrm{~cm}$ (Fig. 1, upper discs). The major component of this toxic activity can be identified as FK520, because isogenic FK520-resistant mutant strains lacking FKBP12 ( $f$ pr 1 ) or expressing a dominant FK520-resistant calcineurin mutant (CMP2-1) were relatively resistant to growth inhibition by the 'S. ascomyceticus' toxin (Fig. 1). In these cases, smaller zones of growth inhibition with radii of $0.35 \mathrm{~cm}$ were observed. Based on these observations, we conclude that ' $S$. ascomyceticus' secretes FK520 at a level sufficient to inhibit the growth of competing yeast cells that are sensitive to FK520. ' $S$. ascomyceticus' secretes an additional toxin and some residual growth inhibition was observed with mutant yeast strains that are resistant to FK506 and FK520 (Fig. 1; Breuder et al., 1994; Hemenway et al., 1995).

Similar results were obtained with an isogenic pair of Streptomyces strains, one that produces FK506 (MA6548) and the second which has a mutation in the FK506 biosynthetic machinery and produces reduced levels of FK506 (Motamedi et al., 1997). As shown in Fig. 1, the wild-type FK506-producing strain inhibited the growth of the FK506-sensitive yeast strain TB23 to yield a modest zone of inhibition with a radius of $0.24 \mathrm{~cm}$, whereas the mutated Streptomyces strain that produces a reduced level of FK506 did not inhibit growth (Fig. 1). Yeast mutants lacking FKBP12 (fpr1) or expressing an FK506-resistant calcineurin mutant (CMP2-1) were resistant to the toxic activity secreted by the wild-type Streptomyces strain (Fig. 1). A control disc containing FK506 inhibited the growth of the wild-type yeast strain $(0.67 \mathrm{~cm}$ radius zone of inhibition) but not of either FK506-resistant mutant yeast strain (Fig. 1).

\section{S. hygroscopicus inhibits Saccharomyces cerevisiae growth via FKBP12-dependent inhibition of TOR}

We performed analogous experiments to test if the rapamycin-producing actinomycete $S$. hygroscopicus also secretes toxins that inhibit yeast growth. Rapamycin potently inhibits the growth of all laboratory yeast strains that have been tested, with an MIC of $\sim 25-100 \mathrm{ng} \mathrm{ml}^{-1}$ (Heitman et al., 1991a). Yeast mutants that are resistant to rapamycin either lack the rapamycin drug-binding protein FKBP12 $(f p r 1)$ or express mutant forms of the FKBP12/rapamycin target 


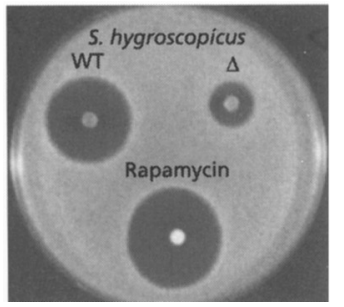

WT

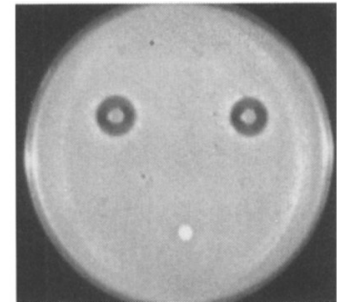

fpr1

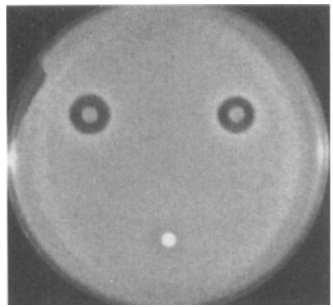

tor1-1

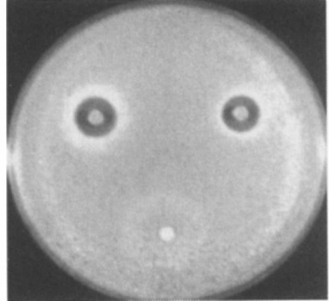

tor2-1

Fig. 2. S. hygroscopicus inhibits growth of wild-type Saccharomyces cerevisiae but not of rapamycin-resistant mutant strains. Cells of isogenic yeast strains expressing wild-type FKBP12 and TOR proteins (WT, JK9-3d), lacking FKBP12 (fpr1, JHY3-3B) or expressing rapamycin-resistant mutant forms of the TOR1 or TOR2 proteins (tor1-1, R1; tor2-1, R17) were resuspended in top agar on the surface of YPD medium, overlaid with inverted agar discs bearing $S$. hygroscopicus rapamycin-producing strain ATCC 29253 (WT, upper left disc), an isogenic S. hygroscopicus mutant strain that does not produce rapamycin (WHM1651; $\Delta$, upper right disc) or control discs containing $1 \mu \mathrm{g}$ rapamycin (lower disc) and incubated at $30^{\circ} \mathrm{C}$ for $48 \mathrm{~h}$.

proteins, the TOR kinase homologues, which do not bind the FKBP12/rapamycin complex as a consequence of mutations in the FKBP12/rapamycin-binding domain on TOR (Cafferkey et al., 1993; Heitman et al., 1991a; Helliwell et al., 1994; Lorenz \& Heitman, 1995; Zheng et al., 1995).

S. bygroscopicus secretes a toxin that potently inhibits the growth of the wild-type, rapamycin-sensitive yeast strain JK9-3d, resulting in a zone of inhibition with a radius of $1.56 \mathrm{~cm}$ (Fig. 2, upper left disc). In contrast, the isogenic rapamycin-resistant yeast mutants lacking FKBP12 ( $p$ pr1) or expressing rapamycin-resistant TOR mutants (tor $1-1$, tor $2-1$ ) were all relatively resistant to growth inhibition by the secreted S. hygroscopicus toxic activity, as indicated by smaller zones of inhibition with radii of $0.56,0.53$ and $0.5 \mathrm{~cm}$ (Fig. 2, upper left disc). Thus, S. hygroscopicus secretes sufficient rapamycin to inhibit the growth of competing rapamycin-sensitive yeast cells. With the isogenic $S$. hygroscopicus mutant strain that does not produce rapamycin, a smaller zone of inhibition (radius $0.5-0.63 \mathrm{~cm}$ ) was observed that was equivalent in size in the rapamycin-sensitive and -resistant yeast strains (Fig. 2, upper right disc). In contrast, a control disc containing rapamycin potently inhibited the wild-type yeast strain (zone of inhibition radius $1.94 \mathrm{~cm}$ ) but had no toxic effect against fpr1, tor 1-1 or tor 2-1 rapamycin-resistant yeast strains (Fig. 2, lower disc). Taken together, these observations indicate that $S$. hygroscopicus secretes both rapamycin and an additional toxin that inhibit growth of yeast.

\section{Streptomyces sp. inhibits C. neoformans growth via FKBP12-dependent inhibition of calcineurin- dependent growth}

To determine if the growth-inhibiting toxins secreted by Streptomyces would affect another micro-organism that is quite distantly related to Saccharomyces cerevisiae, we employed a series of isogenic FK506/rapamycinsensitive and -resistant strains of the pathogenic basidiomycete C. neoformans. As with yeast, growth of $C$. neoformans is inhibited by both FK506 and rapamycin (Odom et al., 1997a). In previous studies, we identified mutations in C. neoformans that confer resistance to FK506 (FKR1-1, mutant strain C21F2), to FK506 and rapamycin (frr1-3, mutant strain $\mathrm{C} 21 \mathrm{~F} 3$ ) or to rapamycin (tor1-1, mutant strain CN111) (Cruz et al., 1999; Odom et al., 1997a). Here we used this set of isogenic strains to further analyse the toxins secreted by Streptomyces sp.

As with Saccharomyces cerevisiae, cells of the wild-type C. neoformans strain JEC21 were resuspended in top agar, supported on rich YPD medium and confronted with competing cells of the wild-type FK506-producing Streptomyces strain MA6548 or an isogenic mutant strain that produces reduced levels of FK506. In previous studies, we found that growth of $C$. neoformans is resistant to the calcineurin inhibitor FK 506 at $24^{\circ} \mathrm{C}$ but markedly sensitive at $37^{\circ} \mathrm{C}$ (Odom et al., 1997a, b). Correspondingly, C. neoformans mutants lacking calcineurin are viable at $24^{\circ} \mathrm{C}$ but not at $37^{\circ} \mathrm{C}$; hence, calcineurin is essential for growth at elevated temperatures in this organism (Odom et al., 1997a).

In accordance with these observations, a toxin secreted by Streptomyces strain MA6548 potently inhibited C. neoformans growth at $37^{\circ} \mathrm{C}$ (halo radius $1.23 \mathrm{~cm}$ ) but not at $30^{\circ} \mathrm{C}$ (Fig. 3, upper left disc). Moreover, the FK506-resistant FKBP12 mutant strain (frr1-3, C21F3) and a dominant FK506-resistant, rapamycin-sensitive mutant strain that is likely to harbour a calcineurin mutation (FKR1-1, C21F2) were completely resistant to growth inhibition by Streptomyces sp. (Fig. 3). In comparison, the zone of inhibition of the wild-type strain was reduced to $0.68 \mathrm{~cm}$ with the Streptomyces mutant strain that produces reduced FK506 and this strain did not inhibit growth of the FK506-resistant C. neoformans strains (Fig. 3, upper right disc). Finally, a control disc containing FK506 potently inhibited the growth of wild-type cells at $37^{\circ} \mathrm{C}$ but not at $30^{\circ} \mathrm{C}$, and did not inhibit growth of the frr1 or FKR1-1 FK506resistant mutant strains (Fig. 3, lower disc). These observations support the conclusion that Streptomyces 

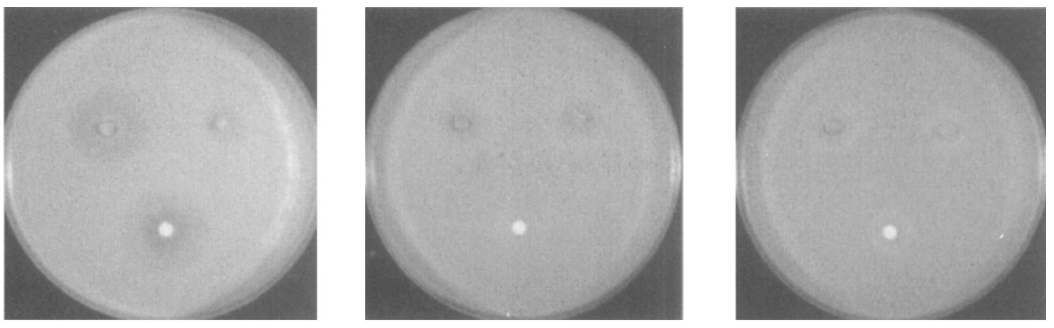

$30^{\circ} \mathrm{C}$

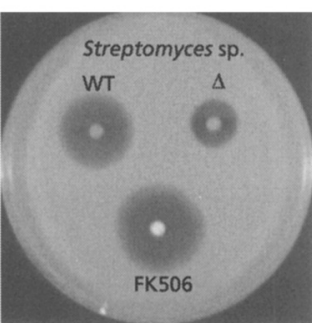

WT

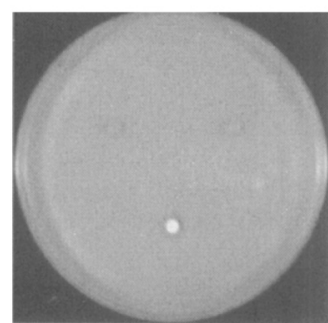

frr1

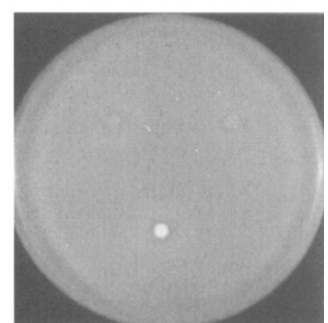

FKR1-1

Fig. 3. Streptomyces sp. secretes a toxin that inhibits growth of $\mathrm{C}$. neoformans at $37{ }^{\circ} \mathrm{C}$ but not at $30{ }^{\circ} \mathrm{C}$ and requires FKBP12 and calcineurin for action. Cells of isogenic C. neoformans strains expressing wild-type FKBP12 and calcineurin proteins (WT, JEC21) or FK506-resistant mutant strains that lack the FKBP12 protein (frr1-3, C21F3) or express the dominant FK506 resistance mutation FKR1-1 (strain C21F2) were resuspended in top agar on the surface of YPD medium. The medium was then overlaid with inverted agar discs bearing Streptomyces sp. FK506-producing strain MA6548 (WT, upper left disc), Streptomyces sp. strain M24 with reduced FK506 production ( $\Delta$, upper right disc) or a control disc with $1 \mu \mathrm{g} \mathrm{FK} 506$ (lower disc) and incubated at 30 or $37^{\circ} \mathrm{C}$ for $48 \mathrm{~h}$.

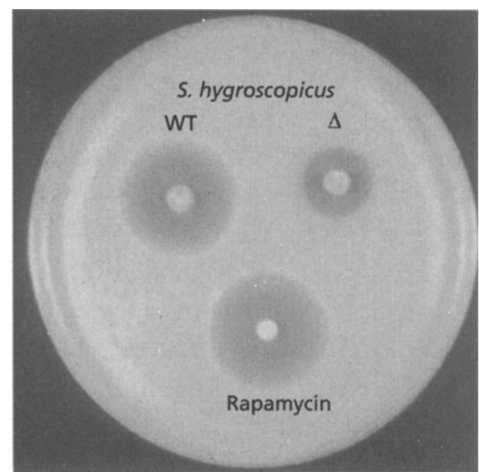

WT

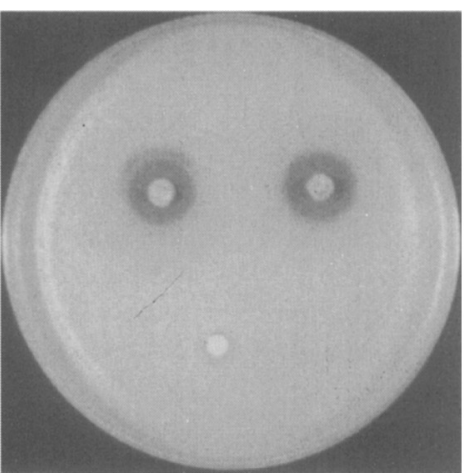

frr1

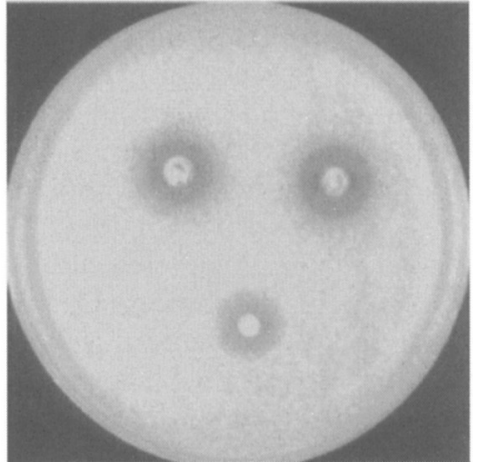

tor1-1

Fig. 4. Rapamycin-resistant $C$. neoformans mutants are resistant to toxins produced by $S$. hygroscopicus. Cells of isogenic C. neoformans strains expressing wild-type FKBP12 and calcineurin proteins (WT, JEC21), an FK506-rapamycin-resistant FKBP12 mutant (frr 1-3, C21F3) and a rapamycin-resistant Tor mutant (tor 1-1, CN111) were resuspended in top agar on the surface of YPD medium, overlaid with an inverted agar disc bearing a rapamycin-producing $S$. hygroscopicus strain (WT, upper left disc), the $S$. hygroscopicus mutant strain that does not produce rapamycin ( $\Delta$, upper right disc) or a control disc containing $1 \mu \mathrm{g}$ rapamycin (lower disc) and incubated at $37^{\circ} \mathrm{C}$ for $48 \mathrm{~h}$.

strain MA6548 secretes sufficient FK506 to inhibit the growth of competing C. neoformans cells via FKBP12dependent inhibition of calcineurin.

\section{S. hygroscopicus inhibits C. neoformans growth via FKBP12-dependent inhibition of TOR}

Analogous experiments were performed to examine inhibition of $C$. neoformans growth by the rapamycinproducing S. hygroscopicus strain ATCC 29253 and an isogenic mutant that does not produce rapamycin (WHM1651). Rapamycin inhibits the growth of wild-type C. neoformans ( $\mathrm{MIC}=\sim 1 \mu \mathrm{g} \mathrm{m} l^{-1}$ ) and rapamycin-resistant mutants lacking FKBP12 or with TOR mutations have been identified (Cruz et al., 1999).

The $S$. bygroscopicus wild-type strain produces a toxin that potently inhibits the growth of the wild-type $C$. neoformans strain JEC21 (zone of inhibition radius $1.45 \mathrm{~cm}$ ) (Fig. 4, upper left disc). In comparison, the 
(a)
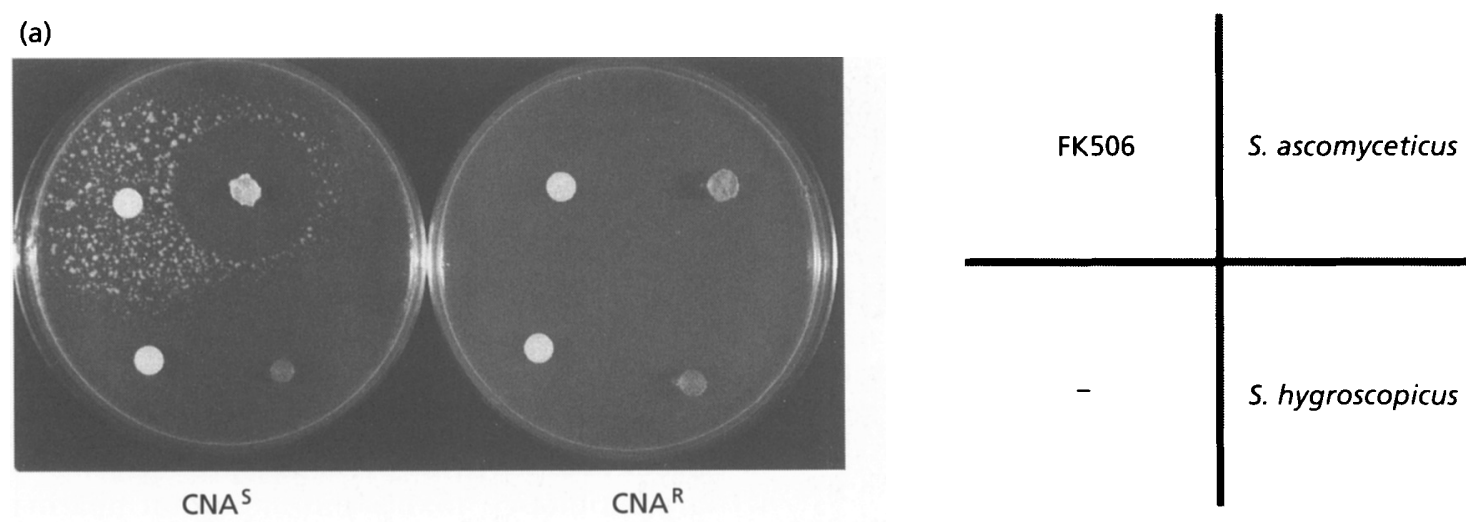

(b)
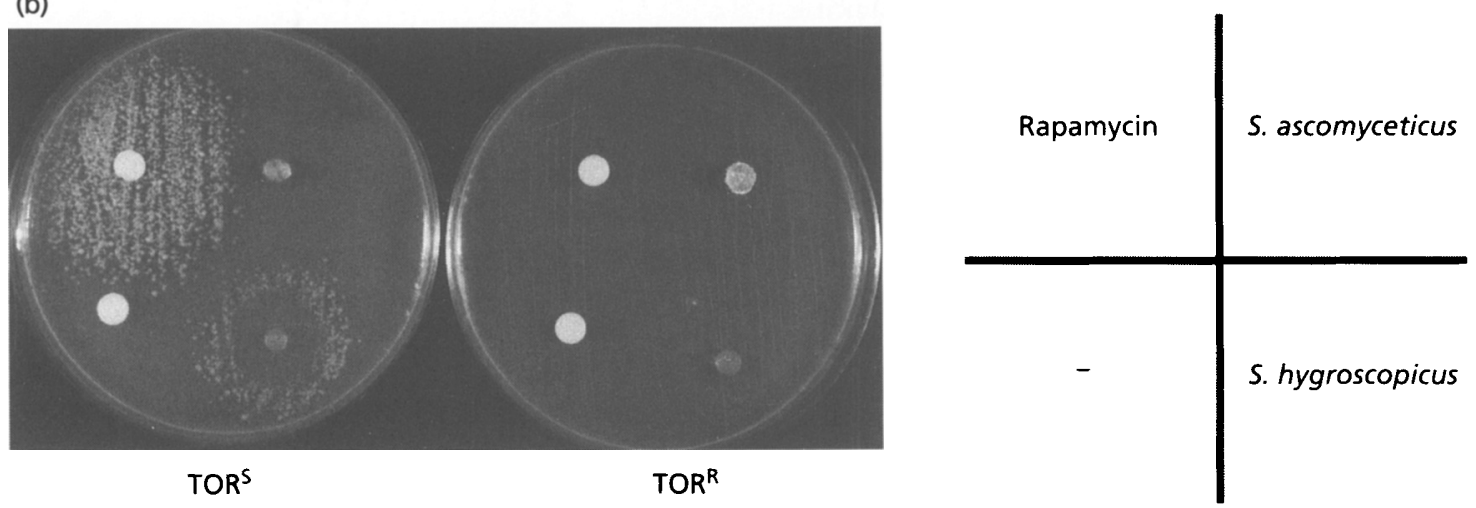

Fig. 5. Streptomyces sp. secretes ligands that promote FKBP12/calcineurin and FKBP12/TOR interactions in the yeast twohybrid assay. (a) Cells of the yeast two-hybrid host strain SMY87-4 (fpr1::hisG) co-expressing the GAL4 DNA-binding domain fused to FKBP12 [GAL4(BD)/FKBP12] and the GAL4 activation domain fused to wild-type calcineurin $A\left(C N A^{5}\right)$ or an FK520-resistant mutant form of calcineurin $A\left(C N A^{R}\right)$ were resuspended in top agar and grown on the surface of medium lacking adenine. Discs bearing FK506 or no drug (-), or agar plugs bearing 'S. ascomyceticus' or $S$. hygroscopicus, were placed on the surface of the agar. Growth indicates GAL4-dependent expression of the GAL-ADE2 reporter gene and results from formation of a GAL4/FKBP12/FK506/FK520/calcineurin/GAL4 complex. (b) Cells of the yeast two-hybrid host strain SMY87-4 (fpr1::hisG) co-expressing the GAL4 activation domain fused to FKBP12 [GAL4(AD)/FKBP12] and the GAL4 DNA-binding domain fused to wild-type TOR (TOR ${ }^{5}$ ) or a rapamycin-resistant mutant form of TOR (TOR ${ }^{R}$ ) were resuspended in top agar and grown on the surface of medium lacking adenine. Discs bearing rapamycin or no drug (-), or agar plugs bearing 'S. ascomyceticus' or S. hygroscopicus, were placed on the surface of the agar. Growth indicates GAL4-dependent expression of the GAL-ADE2 reporter gene and results from formation of a GAL4/FKBP12/rapamycin/TOR/GAL4 complex.

rapamycin-resistant FKBP12 mutant strain C21F3 (frr13) was relatively resistant to the $S$. hygroscopicus toxin, with a smaller zone of inhibition (radius $0.75 \mathrm{~cm}$ ) compared to the isogenic wild-type strain JEC21 (Fig. 4). Similarly, an isogenic mutant strain expressing a TOR1 mutant protein that no longer binds FKBP12/ rapamycin (Cruz et al., 1999) was also relatively resistant to the $S$. hygroscopicus toxin, with a zone of inhibition with a radius of $0.75 \mathrm{~cm}$ (Fig. 4). In accordance with these findings, the zone of growth inhibition with the Streptomyces WHM1651 mutant strain that does not produce rapamycin was reduced to $0.75 \mathrm{~cm}$ in both the rapamycin-sensitive wild-type strain JEC21 and the rapamycin-resistant frr1 and tor $1-1$ mutant strains (Fig. 4, upper right disc). Finally, a control disc containing rapamycin potently inhibited the growth of the wild-type strain (zone of inhibition radius $1.45 \mathrm{~cm}$ ), whereas the frr1 mutant strain was completely resistant to rapamycin and the tor 1-1 mutant strain was largely resistant to rapamycin (Fig. 4, lower disc). Taken together, these observations indicate that $S$. bygroscopicus produces both rapamycin and a second toxin with a different mechanism of action (Fig. 4).

\section{'S. ascomyceticus' toxin mediates FKBP12/calcineurin interaction}

To provide another means to determine if FK520 produced by 'S. ascomyceticus' promotes an interaction between FKBP12 and calcineurin in vivo, we employed the yeast two-hybrid system. Protein-protein and protein-ligand-protein interactions can be monitored in 
the two-hybrid assay by reconstitution of the GAL4 transcriptional activator (Fields \& Song, 1989).

A two-hybrid host strain whose growth is resistant to FK520 (SMY87-4, see Table 1) was transformed with plasmids that express yeast FKBP12 fused to the GAL4 DNA-binding domain [GAL4(BD)/FKBP12] and yeast calcincurin A fused to the GAL4 activation domain $[\mathrm{GAL} 4(\mathrm{AD}) / \mathrm{CNA}]$. Cells were plated in top agar on synthetic medium lacking leucine and tryptophan (to select for the plasmids) and also lacking adenine (as a measure of expression of the $G A L-A D E 2$ reporter gene). The cells were then overlaid with inverted agar discs bearing 'S. ascomyceticus' or a disc containing FK506. A halo of colonies was observed surrounding both the disc bearing ' $S$. ascomyceticus' and the control disc containing FK 506, but not around a control disc lacking FK 506 or an agar disc bearing $S$. hygroscopicus, which secretes rapamycin but not FK520 (Fig. 5a). The presence of growth indicates the formation of a GAL4/FKBP12/ ligand/calcineurin/GAL4 complex that then activates transcription of the $G A L-A D E 2$ reporter gene to support growth in medium lacking adenine. In contrast, no growth was observed when a mutation (W388C, CMP21) that prevents FKBP12/FK506 binding was introduced into the GAL4/calcineurin A fusion protein (Fig. 5a, $\mathrm{CNA}^{\mathrm{R}}$ ). Finally, the finding that a zone of inhibition surrounded the disc of ' $S$. ascomyceticus', but not the control disc with FK506 alone, indicates that ' $S$. ascomyceticus' secretes FK520 and an additional toxin. In summary, these observations support the conclusion that 'S. ascomyceticus' secretes sufficient FK520 to form FKBP12/FK520/calcineurin complexes in competing yeast cells.

\section{S. hygroscopicus toxin mediates cryptococcal FKBP12/TOR interaction}

Analogous experiments were performed using the yeast two-hybrid system to test if $S$. hygroscopicus secretes rapamycin and promotes formation of an FKBP12/ rapamycin/TOR complex. In this case, the yeast twohybrid strain was co-transformed with plasmids that express GAL4(BD)/TOR and GAL4(AD)/FKBP12 fusion proteins. In addition, to render growth of this strain resistant to rapamycin, a third plasmid was introduced that expresses a dominant, rapamycinresistant allele of the yeast TOR 1 protein. Again, growth was readily apparent surrounding the agar disc bearing S. bygroscopicus and around a disc bearing rapamycin, but not around a control disc or an agar disc bearing ' $S$. ascomyceticus' (Fig. 5b). In this assay, growth indicates formation of an FKBP12/rapamycin/TOR complex and GAL4-dependent expression of the GAL-ADE2 reporter gene. In contrast, no growth was observed when a mutation that prevents FKBP12/rapamycin binding to TOR was introduced into the GAL4(BD)/TOR fusion protein (Fig. 5B, TOR ${ }^{\mathrm{R}}$ ). In control experiments, strain WHM 1651, which does not produce rapamycin, did not promote interaction of FKBP12 and TOR in the twohybrid assay (data not shown). These findings support the conclusion that $S$. hygroscopicus secretes rapamycin and promotes the formation of an FKBP12/ rapamycin/TOR complex in adjacent yeast cells. The observation that a zone of growth inhibition is found surrounding the disc of Streptomyces cells, and not around the control disc containing rapamycin alone, again indicates that $S$. bygroscopicus secretes an additional toxin.

\section{DISCUSSION}

Streptomyces and a variety of other micro-organisms produce a vast array of natural products, many of which are antibiotics, toxins and important pharmaceuticals. Here we present evidence that Streptomyces spp. produce the immunosuppressants FK506, FK520 and rapamycin as antimicrobial agents to inhibit the growth of competing micro-organisms (Fig. 6).

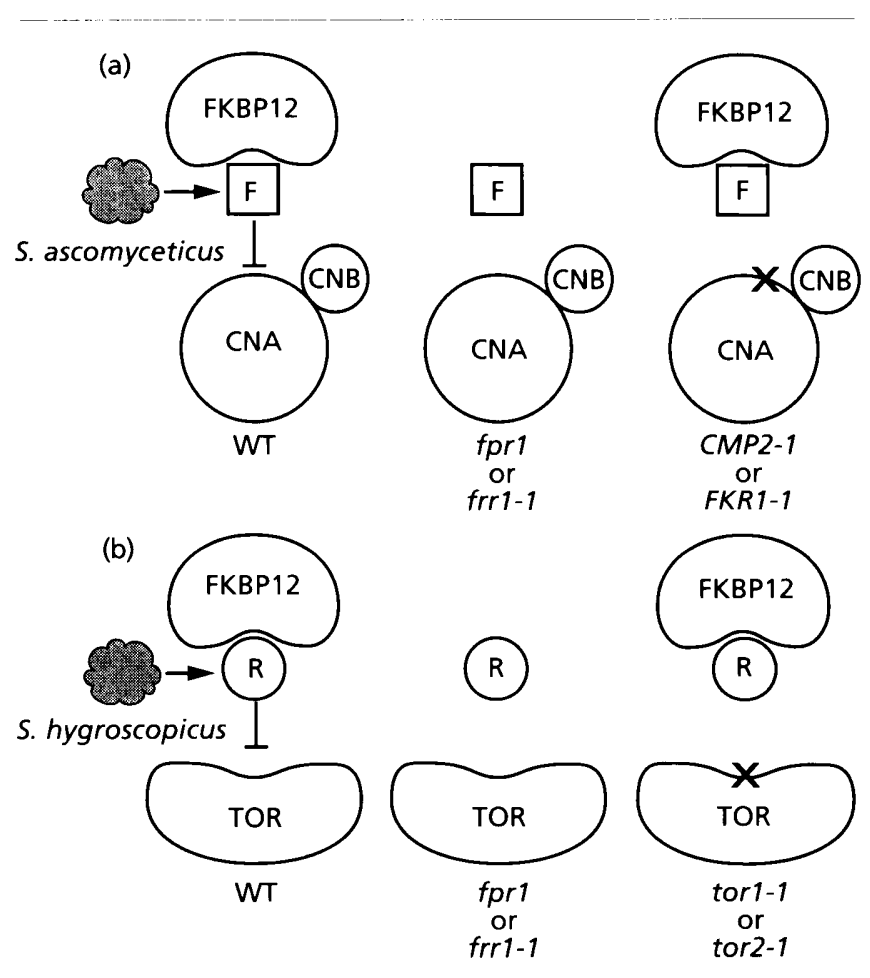

Fig. 6. The antifungal actions of FK506, FK520 and rapamycin are mediated by interactions with the conserved FKBP12, calcineurin and TOR proteins. (a) Streptomyces $\mathrm{sp}$. produces a toxin that inhibits the growth of yeast and fungi. A major component of this toxic activity is FK506 or FK520 (F) which, in a complex with the FKBP12 protein, inhibits the protein phosphatase calcineurin, here indicated as a heterodimer of the catalytic CNA and regulatory CNB subunits. Growth of wild-type yeast and fungi is inhibited by FK506, whereas mutants lacking FKBP12 (fpr1, frr 1-1 or -3) or expressing FK506-resistant calcineurin mutants (CMP2-1, FKR1-1) are drug-resistant. (b) $S$. hygroscopicus produces toxins that inhibit the growth of yeasts and fungi. One major component of this activity is rapamycin (R) which, in complex with the FKBP12 protein, inhibits the TOR kinase homologues. Growth of wild-type yeasts and fungi is inhibited by rapamycin, whereas mutants lacking FKBP12 (fpr 1 , frr1-1 or 3) or expressing rapamycin-resistant TOR mutants (tor 1-1, tor2-1) are rapamycin-resistant. 
Using a series of Saccharomyces cerevisiae and C. neoformans mutant strains with defined mutations that confer sensitivity or resistance to FK506/FK520 and rapamycin, we have demonstrated that the principle components of these toxic activities are indeed FK506, FK520 and rapamycin. These observations are summarized in Fig. 6. First, mutants lacking the FK506/ rapamycin-binding protein FKBP12 are viable and resistant to a toxin produced by ' $S$. ascomyceticus' and S. hygroscopicus. Correspondingly, yeast and $C$. neoformans mutants expressing mutant, drug-resistant forms of the FKBP12/FK506 target protein calcineurin that do not bind FKBP12/FK506 (Cardenas et al., 1995b) are resistant to FK506 produced by Streptomyces. Similarly, yeast and fungal mutants expressing mutant drug-resistant forms of the FKBP12/rapamycin target proteins TOR 1 and TOR 2 in yeast (Lorenz \& Heitman, 1995), or the TOR1 homologue in C. neoformans (Cruz et al., 1999), are resistant to rapamycin produced by $S$. hygroscopicus. Finally, using the yeast two-hybrid system, we have demonstrated that ' $S$. ascomyceticus' secretes a ligand that promotes FKBP12 binding to wildtype calcineurin, but not to a calcineurin mutant that fails to bind FKBP12/FK520, and that S. hygroscopicus secretes a ligand that promotes FKBP12 binding to wildtype TOR, but not to a mutant TOR that fails to bind FKBP12/rapamycin. We conclude that Streptomyces secrete sufficient FK506, FK520 and rapamycin to inhibit the growth of competing, drug-sensitive yeast and fungal cells (Fig. 6).

The mechanisms by which Streptomyces produce these antifungal agents provides further evidence that these compounds may have evolved early and for a specific purpose. FK506 and rapamycin are members of the macrolide family of compounds, which are synthesized by polyketide synthases. In the case of rapamycin, a large array of related biosynthetic enzymes have been characterized that catalyse the sequential steps in the synthesis of the macrolide ring (Aparicio et al., 1996; Molnár et al., 1996; Schwecke et al., 1995). A related series of enzymes is involved in the biosynthesis of FK506 and FK520 (Motamedi et al., 1996; Nielsen et al., 1991). In both cases, a large, complicated series of genes is required to synthesize the natural product. Other macrolides, such as the antibiotic erythromycin, are synthesized by similar, related enzyme clusters (Cortes et al., 1990; Donadio et al., 1991; Katz, 1997). The diversity of compounds produced by related means suggests that these enzymes may have evolved quite early, perhaps prior to the evolution of multicellular eukaryotes and consistent with a role as antimicrobial agents. Many other members of the macrolide family, including erythromycin and streptomycin, are wellknown antibiotics, again suggesting that the role of other members of this family of natural products could serve to inhibit the growth of competing microorganisms. The great complexity of the biosynthetic machinery for these natural products requires that the producing organism invests a great deal of energy in their synthesis, which would again be consistent with a role in growth competition. An alternative hypothesis is that the natural products FK506 and rapamycin might play a signalling role as pheromones, but no evidence to support such a role has as yet been adduced.

A general question that applies to all micro-organisms that make toxins is how the producing organism protects itself from potential suicide. In some cases, such as colicin production in bacteria, the host cell produces an immunity protein that counteracts the lethal action of the toxin. In the case of Streptomyces that produce FK506 and rapamycin, these organisms have been found to express an FKBP12 homologue that binds FK520 and rapamycin with high affinity (Pahl \& Keller, 1992). On the other hand, both $S$. hygroscopicus and ' $S$. ascomyceticus' were found to be resistant to both FK520 and rapamycin (Pahl \& Keller, 1992). One plausible hypothesis is that these bacteria lack the calcineurin and TOR protein targets of FKBP12/drug complexes. Alternatively, calcineurin and TOR homologues might be present but not required for vegetative growth, as is the case with calcineurin in wild-type Saccharomyces cerevisiae strains and may be the case for TOR in Schizosaccharomyces pombe in which vegetative growth is not sensitive to rapamycin (Weisman et al., 1997). Finally, Streptomyces spp. could have evolved mutations in FKBP12, calcineurin or TOR that prevent inhibition by FK506, FK520 and rapamycin.

Why might natural products that target fungal and yeast cells have any activity against mammalian cells, let alone such a specific immunosuppressive action in mammals? The targets of these agents, the FKBP12 prolyl isomerase, the phosphatase calcineurin and the TOR kinase homologues, are highly conserved from yeast to man with $\sim 50 \%$ sequence identity despite $\sim 1$ billion years of evolution. In the case of calcineurin and TOR, one hypothesis is that these proteins play important signal transduction roles involving other conserved signalling molecules and that this, in part, has constrained their evolution. The case of FKBP12 is much more mysterious given that its stable interactions with calcineurin and TOR occur only when FK506 or rapamycin are present. Our previous findings have suggested that these proteins might normally interact under some physiological conditions (see Cardenas et al., 1994). In summary, natural products that evolved to target yeast and fungal growth can have quite specific effects in multicellular eukaryotes by targeting conserved target proteins.

Our studies underscore the highly conserved nature of life and the power of studies on natural product toxins, both as probes of cell function and as a source of valuable pharmaceuticals. These studies may also enable mutagenic approaches to study FK506- and rapamycinproducing organisms and enzymes, and their specificity. For example, our approaches could be readily adapted to screen for mutant bacteria that make toxins that act via FKBP12 but not on calcineurin or TOR. Alterations in culture conditions and biotransformation approaches have been found to result in the production of drug analogues (Khaw et al., 1998; Nishida et al., 1995) and these could be readily screened for activity against novel 
targets using the approaches described here. Finally, novel FKBP12-binding ligands may already exist in nature and in fact two classes of novel FKBP12-binding ligands, meridamycin and the antascomicins, have recently been described (Fehr et al., 1996; Salituro et al., 1995). Although the biological properties of these novel agents remain to be explored, our studies suggest that one role might be as novel antimicrobial agents.

\section{ACKNOWLEDGEMENTS}

We thank Drs C. Richard Hutchinson and Haideh Motamedi for supplying strains of Streptomyces and Ronald Germain for stimulating discussions. Rapamycin was provided by the Drug Discovery branch of the NCI and FK506 by Fujisawa Pharmaceuticals. This research was supported by K01 career development award CA77075 from the NCI (to M.E. C.) and NIAID grants R01 AI39115, AI41937, a supplement to AI41937 (to M.C.C.), and P01 award AI44975 to the Duke University Mycology Research Unit. Joseph Heitman is an associate investigator of the Howard Hughes Medical Institute and a Burroughs Wellcome Scholar in Molecular Pathogenic Mycology.

\section{REFERENCES}

Abraham, R. T. \& Wiederrecht, G. J. (1996). Immunopharmacology of rapamycin. Annu Rev Immunol 14, 483-510.

Alarcon, C. M., Cardenas, M. E. \& Heitman, J. (1996). Mammalian RAFT1 kinase domain provides rapamycin-sensitive TOR function in yeast. Genes Dev' 10, 279-288.

Aparicio, J. F., Molnár, I., Schwecke, T., König, A., Haydock, S. F., Khaw, L. E., Staunton, J. \& Leadlay, P. F. (1996). Organization of the biosynthetic gene cluster for rapamycin in Streptomyces bygroscopicus: analysis of the enzymatic domains in the modular polyketide synthase. Gene 169, 9-16.

Baker, H., Sidorowicz, A., Sehgal, S. N. \& Venzina, C. (1978). Rapamycin (AY-22,989), a new antifungal antibiotic. III. In vitro and in vivo evaluation. J Antibiot 31, 539-545.

Barbet, N. C., Schneider, U., Helliwell, S. B., Stansfield, I., Tuite, M. F. \& Hall, M. N. (1996). TOR controls translation initiation and early (il progression in yeast. Mol Biol Cell 7, 25-42.

Bram, R. J., Hung, D. T., Martin, P. K., Schreiber, S. L. \& Crabtree, G. R. (1993). Identification of the immunophilins capable of mediating inhibition of signal transduction by cyclosporin $\mathrm{A}$ and FK506: roles of calcineurin binding and cellular location. Mol Cell Biol 13, 4760-4769.

Breuder, T., Hemenway, C. S., Movva, N. R., Cardenas, M. E. \& Heitman, J. (1994). Calcineurin is essential in cyclosporin A- and FK.506-sensitive yeast strains. Proc Natl Acad Sci USA 91, $5.372-5376$

Brown, E. J., Albers, M. W., Shin, T. B., Ichikawa, K., Keith, C. T., Lane, W. S. \& Schreiber, S. L. (1994). A mammalian protein targeted by G1-arresting rapamycin-receptor complex. Nature 369, 756-759.

Brunn, G. J., Hudson, C. C., Sekulic, A., Williams, J. M., Hosoi, H., Houghton, P. J., Lawrence, J. C., Jr \& Abraham, R. T. (1997). Phosphorylation of the translational repressor PHAS-I by the mammalian target of rapamycin. Science 277, 99-101.

Cafferkey, R., Young, P. R., McLaughlin, M. M. \& 7 other authors (1993). Dominant missense mutations in a novel yeast protein related to mammalian phosphatidylinositol 3-kinase and VPS34 abrogate rapamycin cytotoxicity. Mol Cell Biol 13, 6012-6023.
Cardenas, M. E., Hemenway, C., Muir, R. S., Ye, R., Fiorentino, D. \& Heitman, J. (1994). Immunophilins interact with calcineurin in the absence of exogenous immunosuppressive ligands. EMBO J 13, 5944-5957.

Cardenas, M. E., Zhu, D. \& Heitman, J. (1995a). Molecular mechanisms of immunosuppression by cyclosporin, FK506, and rapamycin. Curr Opin Nephrol Hypertens 4, 472-477.

Cardenas, M. E., Muir, R. S., Breuder, T. \& Heitman, J. (1995b). Targets of immunophilin-immunosuppressant complexes are distinct highly conserved regions of calcineurin A. EMBO J14, 2772-2783.

Chadwick, D. J. \& Whelan, J. (1992). Secondary Metabolites: Their Function and Euolution. New York: Wiley.

Chiu, M. I., Katz, H. \& Berlin, V. (1994). RAPT1, a mammalian homolog of yeast Tor, interacts with the FKBP12/rapamycin complex. Proc Natl Acad Sci USA 91, 12574-12578.

di Como, C. J. \& Arndt, K. T. (1996). Nutrients, via the Tor proteins, stimulate the association of Tap42 with type $2 \mathrm{~A}$ phosphatases. Genes Dev 10, 1904-1916.

Cortes, J., Haydock, S. H., Roberts, G. A., Bevitt, D. J. \& Leadlay, P. F. (1990). An unusually large multifunctional polypeptide in the erythromycin producing polyketide synthase of Saccharopolyspora erythraea. Nature 348, 176-178.

Cruz, M. C., Cavallo, L. M., Görlach, J. M., Cox, G., Perfect, J. R., Cardenas, M. E. \& Heitman, J. (1999). Rapamycin antifungal action is mediated via conserved complexes with FKBP12 and TOR kinase homologs in Cryptococcus neoformans. Mol Cell Biol 19, 4101-4112.

Donadio, S., Staver, M. J., McAlpine, J. B., Swanson, S. J. \& Katz, L. (1991). Modular organization of genes required for complex polyketide biosynthesis. Science 252, 675-679.

Douglas, C. M., Foor, F., Marrinan, J. A. \& 11 other authors (1994). The Saccharomyces cerevisiae FKS1 (ETG1) gene encodes an integral membrane protein which is a subunit of $1,3-\beta$-D-glucan synthase. Proc Natl Acad Sci USA 91, 12907-12911.

Fehr, T., Sanglier, J. J., Schuler, W., Gschwind, L., Ponelle, M., Schilling, W. \& Wioland, C. (1996). Antascomicins A, B, C, D and E. Novel FKBP12 binding compounds from a Micromonospora strain. J Antibiot 49, 230-233.

Fields, S. \& Song, O.-K. (1989). A novel genetic system to detect protein-protein interactions. Nature 340, 245-246.

Foor, F., Parent, S. A., Morin, N., Dahl, A. M., Ramadan, N., Chrebet, G., Bostian, K. A. \& Nielsen, J. B. (1992). Calcineurin mediates inhibition by FK506 and cyclosporin of recovery from $\alpha$ factor arrest in yeast. Nature 360, 682-684.

Hatanaka, H., Iwami, M., Kino, T., Goto, T. \& Okuhara, M. (1988a). FR-900520 and FR-900523, novel immunosuppressants isolated from a Streptomyces. I. Taxonomy of the producing strain. $J$ Antibiot 41, 1586-1591.

Hatanaka, H., Kino, T., Miyata, S., Inamura, N., Kuroda, A., Goto, T., Tanaka, H. \& Okuhara, M. (1988b). FR-900520 and FR-900523, novel immunosuppressants isolated from a Streptomyces. II. Fermentation, isolation and physico-chemical and biological characteristics. J Antibiot 41, 1592-1601.

Heitman, J., Movva, N. R. \& Hall, M. N. (1991a). Targets for cell cycle arrest by the immunosuppressant rapamycin in yeast. Science 253, 905-909.

Heitman, J., Movva, N. R., Hiestand, P. C. \& Hall, M. N. (1991b). FK506-binding protein proline rotamase is a target for the immunosuppressive agent FK506 in Saccharomyces cerevisiae. Proc Natl Acad Sci USA 88, 1948-1952.

Heitman, J., Movva, N. R. \& Hall, M. N. (1992). Proline isomerases 
at the crossroads of protein folding, signal transduction, and immunosuppression. New Biol 4, 448-460.

Helliwell, S. B., Wagner, P., Kunz, J., Deuter-Reinhard, M., Henriquez, R. \& Hall, M. N. (1994). TOR1 and TOR2 are structurally and functionally similar but not identical phosphatidylinositol kinase homologues in yeast. Mol Biol Cell 5, 105-118.

Hemenway, C. S., Dolinski, K., Cardenas, M. E., Hiller, M. A., Jones, E. W. \& Heitman, J. (1995). vph6 mutants of Saccharomyces cerevisiae require calcineurin for growth and are defective in vacuolar $\mathrm{H}^{+}$-ATPase assembly. Genetics $141,833-844$.

High, K. P. (1994). The antimicrobial activities of cyclosporine, FK506, and rapamycin. Transplantation 57, 1689-1700.

James, P., Halladay, J. \& Craig, E. A. (1996). Genomic libraries and a host strain designed for highly efficient two-hybrid selection in yeast. Genetics 144, 1425-1436.

Katz, L. (1997). Manipulation of modular polyketide synthase. Chem Rev 97, 2557-2575.

Khaw, L. E., Böhm, G. A., Metcalfe, S., Staunton, J. \& Leadlay, P. F. (1998). Mutational biosynthesis of novel rapamycins by a strain of Streptomyces hygroscopicus NRRL 5491 disrupted in rapL, encoding a putative lysine cyclodeaminase. J Bacteriol $\mathbf{1 8 0}$, 809-814.

Kino, T., Hatanaka, H., Miyata, S. \& 8 other authors (1987). FK506, a novel immunosuppressant isolated from a Streptomyces. II. Immunosuppressive effect of FK-506 in vitro. I Antibiot 40 , 1256-1265.

Kunz, J., Henriquez, R., Schneider, U., Deuter-Reinhard, M., Movva, N. R. \& Hall, M. N. (1993). Target of rapamycin in yeast, TOR2, is an essential phosphatidylinositol kinase homolog required for $G_{1}$ progression. Cell 73, 585-596.

Liu, J., Farmer, J. D., Lane, W. S., Friedman, J., Weissman, I. \& Schreiber, S. L. (1991). Calcineurin is a common target of cyclophilin-cyclosporin A and FKBP-FK506 complexes. Cell 66, 807-815.

Lomovskaya, N., Fonstein, L., Ruan, X., Stassi, D., Katz, L. \& Hutchinson, C. R. (1997). Gene disruption and replacement in the rapamycin-producing Streptomyces hygroscopicus strain ATCC 29253. Microbiology 143, 875-883.

Lorenz, M. C. \& Heitman, J. (1995). TOR mutations confer rapamycin resistance by preventing interaction with FKBP12rapamycin. J Biol Chem 270, 27531-27537.

Luengo, J. I., Yamashita, D. S., Dunnington, D. \& 18 other authors (1995). Structure-activity studies of rapamycin analogs: evidence that the C-7 methoxy group is part of the effector domain and positioned at the FKBP12-FRAP interface. Chem Biol 2, 471-481.

McCabe, R. E., Luft, B. J. \& Remington, J. S. (1986). The effects of cyclosporine on Toxoplasma gondii in vivo and in vitro. Transplantation 41, 611-615.

Molnár, I., Aparicio, J. F., Haydock, S. F., Khaw, L. E., Schwecke, T., König, A., Staunton, J. \& Leadlay, P. F. (1996). Organisation of the biosynthetic gene cluster for rapamycin in Streptomyces bygroscopicus: analysis of genes flanking the polyketide synthase. Gene 169, 1-7.

Moore, T. D. E. \& Edman, J. C. (1993). The $\alpha$-mating type locus of Cryptococcus neoformans contains a peptide pheromone gene. Mol Cell Biol 13, 1962-1970.

Morisaki, M. \& Arai, T. (1992). Identity of immunosuppressant FR-900520 with ascomycin. J Antibiot 45, 126-128.

Motamedi, H., Shafiee, A., Cai, S.-J., Streicher, S. L., Arison, B. H. \& Miller, R. R. (1996). Characterization of methyltransferase and hydroxylase genes involved in the biosynthesis of the immunosuppressants FK506 and FK520. J Bacteriol 178, 5243-5248.

Motamedi, H., Cai, S.-J., Shafiee, A. \& Elliston, K. O. (1997). Structural organization of a multifunctional polyketide synthase involved in the biosynthesis of the macrolide immunosuppressant FK506. Eur J Biochem 244, 74-79.

Nielsen, J. B., Hsu, M.-J., Byrne, K. M. \& Kaplan, L. (1991). Biosynthesis of the immunosuppressant immunomycin: the enzymology of pipecolate incorporation. Biochemistry $\mathbf{3 0}$, 5789-5796.

Nishida, H., Sakakibara, T., Aoki, F. \& 9 other authors (1995). Generation of novel rapamycin structures by microbial manipulations. J Antibiot 48, 657-666.

Odom, A., Muir, S., Lim, E., Toffaletti, D. L., Perfect, J. \& Heitman, J. (1997a). Calcineurin is required for virulence of Cryptococcus neoformans. EMBO J 16, 2576-2589.

Odom, A., Poeta, M. D., Perfect, J. \& Heitman, J. (1997b). The immunosuppressant FK506 and its nonimmunosuppressive analog L-685,818 are toxic to Cryptococcus neoformans by inhibition of a common target protein. Antimicrob Agents Chemother 41, 156-161.

Pahl, A. \& Keller, U. (1992). FK-506-binding proteins from Streptomycetes producing immunosuppressive macrolactones of the FK-506 type. J Bacteriol 174, 5888-5894.

Parent, S. A., Nielsen, J. B., Morin, N., Chrebet, G., Ramadan, N., Dahl, A. M., Hsu, M.-J., Bostian, K. A. \& Foor, F. (1993). Calcineurin-dependent growth of an FK506- and CsA-hypersensitive mutant of Saccharomyces cerevisiae. J Gen Microbiol 139, 2973-2984.

Sabatini, D. M., Erdjument-Bromage, H., Lui, M., Tempst, P. \& Snyder, S. H. (1994). RAFT1: a mammalian protein that binds to FKBP12 in a rapamycin-dependent fashion and is homologous to yeast TORs. Cell 78, 35-43.

Sabers, C. J., Martin, M. M., Brunn, G. J., Williams, J. M., Dumont, F. J., Wiederrecht, G. \& Abraham, R. T. (1995). Isolation of a protein target of the FKBP12-rapamycin complex in mammalian cells. J Biol Chem 270, 815-822.

Salituro, G. M., Zink, D. L., Dahl, A., Nielsen, J., Wu, E., Huang, L., Kastner, C. \& Dumont, F. J. (1995). Meridamycin: a novel nonimmunosuppressive FKBP12 ligand from Streptomyces bygroscopicus. Tetrabedron Lett 36, 997-1000.

Schreiber, S. L. \& Crabtree, G. R. (1992). The mechanism of action of cyclosporin A and FK506. Immunol Today 13, 136-142.

Schwecke, T., Aparicio, J. F., Molnár \& 10 other authors (1995). The biosynthetic gene cluster for the polyketide immunosuppressant rapamycin. Proc Natl Acad Sci USA 92, 7839-7843.

Sehgal, S. N., Baker, H. \& Vezina, C. (1975). Rapamycin (AY22,989), a new antifungal antibiotic. II. Fermentation, isolation and characterization. J Antibiot 28, 727-732.

Sherman, F. (1991). Getting started with yeast. Methods Enzymol 194, 3-21.

Singh, K., Sun, S. \& Vezina, C. (1979). Rapamycin (AY-22,989), a new antifungal antibiotic. IV. Mechanism of action. J Antibiot XXXII, 630-645.

Tropschug, M., Barthelmess, I. B. \& Neupert, W. (1989). Sensitivity to cyclosporin A is mediated by cyclophilin in Neurospora crassa and Sacharomyces cerevisiae. Nature 342, 953-955.

Vezina, C., Kudelski, A. \& Sehgal, S. N. (1975). Rapamycin (AY22,989), a new antifungal antibiotic. I. Taxonomy of the producing Streptomycete and isolation of the active principle. $J$ Antibiot 28, 721-726. 
Weisman, R., Choder, M. \& Koltin, Y. (1997). Rapamycin specifically interferes with the developmental response of fission yeast to starvation. J Bacteriol 179, 6325-6334.

Wong, G. K., Griffith, S., Kojima, I. \& Demain, A. L. (1998). Antifungal activities of rapamycin and its derivatives, prolylrapamycin, 32-desmethylrapamycin, and 32-desmethoxyrapamycin. J Antibiot 51, 487-491.
Zheng, X.-F., Fiorentino, D., Chen, J., Crabtree, G. R. \& Schreiber, S. L. (1995). TOR kinase domains are required for two distinct functions, only one of which is inhibited by rapamycin. Cell $\mathbf{8 2}$, 121-130.

Received 12 April 1999; accepted 23 April 1999. 